\title{
A Review of Machine Learning Models for Predicting Autism Spectrum Disorder
}

\author{
${ }^{* 1} P$. Kanchanamala, ${ }^{2}$ G. Leela Sagar \\ Department of Information Technology, GMR Institute of Technology, Rajam \\ Email: kanchanamala.p@gmrit.org
}

Received: $2^{\text {th }}$ October 2018, Accepted: $2^{\text {th }}$ January 2019, Published: $28^{\text {th }}$ February 2019

\begin{abstract}
Autism spectrum disorder (ASD) is a neurological and developmental disorder that impacts the behavior of the person throughout a life. Every individual with ASD exhibits the difficulty in communication and social interaction with restricted interests and repetitive behaviors. There is no standard diagnosis and treatment for ASD. The social behavior of the children can be improved by early identification of autism spectrum disorder (ASD). A vigorous predictive mechanism desires features such as facial expressions, eye movement, and brain activity images. The research embody diverse approaches to classify and categorize cognitive disorders affected children based on fMRI scan images, facial expressions, gage contingent eye tracking and stereotypical motor movement. Predictive models can be developed to determine if the new patients are developing with ASD using historical patient data. This paper explores the machine learning models built using structured clinical patient data for predicting the ASD subjects.
\end{abstract}

\section{Keywords}

Autism Spectrum Disorder, rs-fMRI, Stereotypical Motor Movements, Machine Learning, Deep Learning

\section{Introduction}

This paper aims to review the various approaches considered and the machine learning models built for early prediction of the Autism Spectrum Disorder (ASD). ASD is a brain development disorder that limits the natural growth of the children in terms of communication and social behaviors [1]. The American Psychiatric Association has created a guide, Diagnostic and Statistical Manual of Mental Disorders (DSM-5), to diagnose mental disorders. As per DSM5 people with ASD suffers with restricted interests, repetitive behaviors which limits them with communication and social interaction difficulties [2]. Due to lack of awareness on the disorder parents ignore the early signs that are exhibited by their children. Hence it is very much necessary for the early prediction of the disorder [3].

Early diagnosis of ASD within the first few years of life allows for treatment while the child's brain is still rapidly developing. Autism spectrum disorder (ASD) diagnoses are on the rise, due in part to physicians adopting more standardized methods for evaluation and diagnosis. Due to lack of standard diagnosing methods and treatment it is difficult to diagnosis ASD. Doctors observe the child's behavior and development to make a diagnosis which needs a lot of time and effort to detect ASD in an individual.

Machine learning is a mathematical learning framework for extracting useful patterns from the available large amounts of data. Extracted knowledge can be helpful to humans in clinical decision making. This computational learning methodologies are being rapidly used in the study of neurocognitive disorders [4].

\footnotetext{
Methodology

We reviewed the literature related to the machine learning models focusing on the prediction of Autism Spectrum Disorder. There are many methodologies to predict the Autism Spectrum Disorder with different input parameters and prediction models. Each methodology focuses on particular input parameter for modeling.

1. Eye Tracking

People with ASD look more at objects, images with more saliency, videos rather than at people. Eye gaze analysis can be used as a tool for diagnosing the people with ASD [5, 23].

S. Wang et al., 2015 considered twenty ASD diagnosed people and nineteen healthy subjects with, mean age of $32.3 \pm 10.4$ years for experiment. For all these people a large Object and Semantic Images and Eye-tracking (OSIE) dataset with eye-tracking data was collected using Tobii TX300 Eye Tracker which collects gaze data at $300 \mathrm{~Hz}$ [5][6]. 700 images from the OSIE [6] database with three pixel-level attributes (color, intensity, and orientation), five objectlevel attributes (size, complexity, convexity, solidity, and eccentricity), and 12 semantic attributes (face, emotion, touched, gazed, motion, sound, smell, taste, touch, text, watchability, and operability) were observed by the Subjects. The images were helpful in understanding different aspects of visual attention, from low-level feature contrasts to high-level semantics. These features were used to train a linear SVM to classify the two populations. Average of all
} 
the features across all trials was used to represent the attention patterns of each eye. The SVM classifier classified the data with an accuracy of $81 \%$ [5].

Children and adults with ASD shows less attention to faces compared to the typically developed (TD) controls [7]. Wenbo Liu et al., have conducted the study on 20 children with ASD and 21 age-matched Typically Developed children and 20 IQ-matched Typically Developed children for the study. Three female faces were used as targets among the twelve Chinese adult female photos displayed to the subjects. The faces were normalized such that majority of facial features were positioned approximately in the same face regions. Area of Interest (AOI) approach was used to analyze the eye movement data in comparing the fixation duration within each predefined face region (e.g. eyes, nose, and mouth) [8]. The distributions of fixation coordinates were selected as a feature. The features were represented using the facial region partitioning and the histogram feature extraction. K- means algorithm was implemented for the Quantization of fixation coordinates. The quantization partitioned the face images into K different cell-like regions which indicate the close proximity in the visual attention location [9]. The labeled histograms were used to train the SVM classifier [10,11]. The SVM classifier classified the data based on the extracted histograms with an accuracy of $88.51 \%$.

\section{Brain Imaging Data:}

Functional Magnetic Resonance Imaging (fMRI) is a useful tool for the prediction of Autism Spectrum Disorder. fMRI is used to measure the brain activity by detecting changes in the blood flow[12]. The neuronal activation is depends on cerebral blood flow. When an area of the brain is in use, blood flow to that region also increases [13]. fMRI uses Blood Oxygen Level Dependent (BOLD) contrast to map neuronal activity in the brain of humans or other animals by imaging the change in the blood flow related to the energy used by the brain cells. Functional magnetic resonance imaging (fMRI) is popular in predicting ASD because of its excellent contrast properties, spatial resolution, and temporal resolution [14].

Omar Dekhil et al., have conducted an experiment on 202 children among which 78 were suffering with ASD and 124 are healthy controls. Neuro images were obtained from the National Database for Autism. Probabilistic Independent Component Analysis (PICA) algorithm was implemented to factorize fMRI scans into spatial and temporal components. From the temporal components, power spectral densities (PSD) were used as features for the diagnosis. 34 PSD components that are highly correlated with 34 atlas areas were selected from all the output components from PICA. Auto encoders followed by probabilistic support vector machine were used for diagnosis. The system was tested using leave one subject out approach and with an accuracy, sensitivity, specificity and area under Receiver Operating Curve (ROC) of $0.985,0.974,0.991$ and 0.998 respectively. The system robustness and diagnosis integrity were ensured using 2 fold, 4 fold and 10 fold cross validation techniques with an accuracy of $81 \%, 82 \%, 84 \%$ [15].

E. Feczko et al., have identified more homogeneous ASD sub groups using a Random Forests approach. Resting state fMRI data was used for constructing a series of decision trees using RF algorithm. A boot strapped data set was generated from a subset of training data per each tree. The group classification was predicted using a subset of features were used to predict. The classification and regression were performed by using Gini impurity and mean square error as the cost functions respectively. The performance of the RF models was assessed by running the RF algorithm in 1000 iterations. 60 percent of participants formed the training dataset and the remaining 40 percent formed the testing dataset per each iteration. Applying the RF algorithm on behavioral data from 7 different tasks ( 34 variables) achieved an overall classification accuracy of $73 \%$ [16].

Anibal Solon Heinsfeld et al., have considered rs-fMRI data of 505 ASD individuals and 530 matched typical controls (TC) for the study. Correlation was calculated for the average of the time series of the regions of interest to investigate the brain connectivity and built the connectivity matrix.

Functional connectivity was used as a feature for the classification of ASD and Typical controls. Predictive model was trained using deionized auto encoders. Deep Neural Network (DNN) has classified the data with an accuracy of $70 \%[17]$.

\section{Stereotypical Motor Movements:}

Children with ASD feels difficulty in social communication and exhibit repetitive behaviors known as stereotypical motor movements (SMM). SMMs include repetitive body rocking, hand flapping, mouthing, and complex finger movements which interfere with social interactions [18]. SMMs can be measured either by interviewing, observing the behaviors directly and through recorded videos. All these methods suffer with the difficulty of measuring the duration and intensity of SMMs. Video based approach is accurate but it is a time consuming process. Instead to reduce the time consumption accelerometer sensors can be employed to measure the SMMs over a period of time. [19]. Matthew S. Goodwin et al., have used wearable wireless sensors to obtain the 3-axes accelerometer data. SMM data classified using Support Vector Machine and Decision Tree with different feature sets based on time-frequency characteristics of accelerometer data [20]. Nastaran Mohammadian Rad et al., have proposed a Deep Neural Network 
with two CNN layers and one pooling layer to classify ASD children using accelerometer data with an accuracy of $82 \%[21]$.

Goodwin, M.S., Haghighi et al., have conducted a longitudinal study on six autism subjects. The data was collected in laboratory and classroom environments. The raw feature space signals were used as the input to a Support Vector Machine (SVM) classifier. Extracted a features including time, frequency, and Stock well transform features were used to evaluate the detection performance. A CNN architecture with randomized initial weights and biases was used to learn a middle representation of the multi-sensor signal. The random initialization of weights and stochastic gradient descent algorithm for optimization, results were different from one training run to another. So, the whole procedure of learning and classification was run 10 times for better optimization. This method has achieved an accuracy of $82 \%$ [22].

\section{Summary}

\begin{tabular}{|c|c|c|c|c|}
\hline Input parameter & Methodology & Accuracy in $\%$ & Ref. & Conclusion \\
\hline \multirow{2}{*}{ Eye tracking } & SVM & 81 & [5] & $\begin{array}{l}\text { ASD people had more fixation to the center of } \\
\text { the image exhibits more attention towards a } \\
\text { particular object in natural images. }\end{array}$ \\
\hline & SVM & 88.51 & [8] & $\begin{array}{l}\text { ASD subjects shows less attention to human } \\
\text { faces and feels difficulty in interpreting human } \\
\text { emotions. }\end{array}$ \\
\hline \multirow{3}{*}{ Brain Imaging Data } & Auto Encoder & 84 & [15] & $\begin{array}{l}\text { Functional connectivity spatial maps were used } \\
\text { for classifying the ASD to TD and improved the } \\
\text { classifier accuracy. }\end{array}$ \\
\hline & $\begin{array}{l}\mathrm{RF} \text { algorithm } \\
\text { With task based } \\
\text { images }\end{array}$ & 73 & [16] & $\begin{array}{l}\text { The behavioral data of subjects from } 7 \text { different } \\
\text { tasks were analyzed. RF model has achieved an } \\
\text { overall classification accuracy of } 73 \% \text { in } \\
\text { classifying the cognitive patterns of individuals } \\
\text { with ASD and without. }\end{array}$ \\
\hline & DNN & 70 & [17] & $\begin{array}{l}\text { The correlation Patterns of posterior network } \\
\text { of brain areas which are activated highly during } \\
\text { rs-AMRI were the most relevant features to the } \\
\text { ASD classification. }\end{array}$ \\
\hline \multirow[b]{2}{*}{$\begin{array}{l}\text { Stereotypical } \\
\text { Movements (SMM) }\end{array}$} & DNN & 82 & [21] & $\begin{array}{l}\text { The classification performance can be } \\
\text { improved with better feature } \\
\text { extraction/learning for detecting SMMs. } \\
\text { Ensemble of LSTMs provide more reliable } \\
\text { SMM detector than every single LSTM. }\end{array}$ \\
\hline & $\begin{array}{l}\text { SVM with CNN for } \\
\text { feature extraction }\end{array}$ & 82 & {$[22]$} & $\begin{array}{l}\text { SMMs are measured by analyzing hand } \\
\text { flapping, body rocking, and simultaneous hand } \\
\text { flapping and body rocking. SMMs a real-time, } \\
\text { person-dependent, adaptive To automate the } \\
\text { measure of algorithms are needed. }\end{array}$ \\
\hline
\end{tabular}




\section{Conclusion}

The Centers for Disease Control and Prevention (CDC) estimates autism's prevalence as 1 in 59 children which includes 1 in 37 boys and 1 in 151 girls. Early identification of ASD is essential to improve the child's ability to learn new skills. Manual diagnosis of the ASD is a much time consuming process for the collection as well as identifying the disorder. The use of Machine learning methods reduces the time and man power and increases the accuracy in detecting the ASD from Typically Developed Controls (TC). This study reviews the machine learning models used to classify the ASD subjects and TC using various input parameters viz. Stereotypical Motor movements, Eye Tracking, Brain Images.

\section{References}

[1] P. K, S. Srinath, S. Seshadri, S. Girimaji, and J. Kommu. "Lost time: Need for more awareness in early intervention of autism spectrum disorder." Asian Journal of Psychiatry, 25:13-15, 2017.

[2] https://www.psychiatry.org/psychiatrists/practice/dsm

[3] Anurekha G and Geetha P," Performance Analysis of Supervised Approaches for Autism Spectrum Disorder Detection." International Journal of Trend in Research and Development (IJTRD),RIET-2017.

[4] Daniel Bone, Matthew S. Goodwin, Matthew P. Black, Chi-Chun Lee, Kartik Audhkhasi, and Shrikanth Narayanan, "Applying Machine Learning to Facilitate Autism Diagnostics: Pitfalls and promises." Journal of Autism Developmental Disorders. 2015 May ; 45(5): 1121-1136. doi:10.1007/s10803-014-2268-6.

[5] S. Wang, M. Jiang, X. M. M. Duchesne, E. A. A. Laugeson, D. P. P. Kennedy, R. Adolphs, and Q. Zhao. "Atypical visual saliency in autism spectrum disorder quantified through model-based eye tracking. Neuron", 88(3):604-616, 2015. 2, 3, 6, 7

[6] J. Xu, M. Jiang, S. Wang, M. S. Kankanhalli, and Q. Zhao. "Predicting human gaze beyond pixels." Journal of Vision, 14(1):28, 2014. 3, 7

[7] T. Falck-Ytter and C. von Hofsten, "How special is social looking in ASD: a review." Progress in brain research, no. 189, pp. 209-22, 2011.

[8] Wenbo Liu, Ming Li, and Li Yi "Identifying Children with Autism Spectrum Disorder Based on Their Face Processing Abnormality: A Machine Learning Framework.”, Autism Research, 2016 Aug;9(8):888-98. doi: 10.1002/aur.1615. Epub 2016 Apr 1.

[9] Yi, L., Feng, C., Quinn, P. C., Ding, H., Li, J., Liu, Y., et al. (2014). “ Do Individuals with and without Autism Spectrum Disorder Scan Faces Differently? A New Multi-Method Look at an Existing Controversy". Autism Research, 7, 72-83.

[10] Cortes, C. and Vapnik, V. (1995) Support-Vector Networks. Machine Learning, 20, 273-297. http://dx.doi.org/10.1007/BF00994018

[11] Chapelle, O., Haffner, P., \& Vapnik, V. N. (1999). "Support vector machines for histogram-based image classification". IEEE Transactions on Neural Networks, 10, 1055-1064.

[12] "Magnetic Resonance, a critical peer-reviewed introduction; functional MRI". European Magnetic Resonance Forum. Retrieved 17 November 2014.

[13] Logothetis, N. K.; Pauls, Jon; Auguth, M.; Trinath, T.; Oeltermann, A. (July 2001). "A neurophysiological investigation of the basis of the BOLD signal in fMRI". Nature. 412 (6843): 150-157. doi:10.1038/35084005. PMID 11449264.

[14] Huettel, S. A.; Song, A. W.; McCarthy, G. (2009), "Functional Magnetic Resonance Imaging (2 ed.)", Massachusetts: Sinauer, ISBN 978-0-87893-286-3

[15] Omar Dekhil, Hassan Hajjdiab, Babajide Ayinde, Ahmed Shalaby, Andy Switala, Dawn Sosnin, Aliaa Elshamekh, Mohamed Ghazal, Robert Keynton, Gregory Barnes, Ayman El-Baz, "Using Resting State Functional MRI to Build A Personalized Autism Diagnosis System”. 2018, https://doi.org/10.1371/journal.pone.0206351.

[16] E. Feczko, N. Balba, O. Miranda-Dominguez, M. Cordova, S.L. Karalunas, L. Irwin, D.V. Demeter, A.P. Hill, B.H. Langhorst, J. Grieser Painter, J. Van Santen, E.J. Fombonne, J.L. Nigg, D.A. Fair "Subtyping cognitive profiles in Autism Spectrum Disorder using a random forest algorithm", Neuro Image, April 2017.

[17] Anibal Solon Heinsfeld, Alexandre Rosa Francob, R. Cameron Craddock, Augusto Buchweit, Felipe Meneguzzia, "Identification of autism spectrum disorder using deep learning and the ABIDE dataset", Neuro Image: Clinical, Volume 17, 2018, Pages 16-23.

[18] LaGrow, S.J. and Repp, A.C. Stereotypic responding: "A review of intervention research.” American Journal of Mental Deficiency 88, 6 (1984), 595-609.

[19] Nastaran Mohammadian Rad, Cesare Furlanello, "Applying Deep Learning to Stereotypical Motor Movement Detection in Autism Spectrum Disorders", 2016 IEEE 16th International Conference on Data Mining Workshops, 2375-9259/16, DOI 10.1109/ICDMW.2016.184 
[20] Matthew S. Goodwin, Marzieh Haghighi, Qu Tang, Murat Akcakaya, Deniz Erdogmus, Stephen Intille, "Moving Towards a Real-Time System for Automatically Recognizing Stereotypical Motor Movements in Individuals on the Autism Spectrum Using Wireless Accelerometry", UBICOMP '14, SEPTEMBER 13 - 17, 2014, SEATTLE, WA, USA.

[21] Nastaran Mohammadian Rad ,Seyed Mostafa Kia, Calogero Zarbo1, Twan van Laarhoven, Giuseppe Jurman1, Paola Venuti, Elena Marchiori, Cesare Furlanello, "Deep Learning for Automatic Stereotypical Motor Movement Detection using Wearable Sensors in Autism Spectrum Disorder", Signal Processing, Volume 144, March 2018, Pages $180-191$

[22] M. S. Goodwin, M. Haghighi, Q. Tang, M. Akcakaya, D. Erdogmus, S. Intille, "Moving towards a real-time system for automatically recognizing stereotypical motor movements in individuals on the autism spectrum using wireless accelerometry", Proceedings of the 2014 ACM International Joint Conference on Pervasive and Ubiquitous Computing, ACM, 2014, pp. 861-872.

[23] https://strammer.com/en/eye-test-autism/ 